\title{
Elektrische Sondenmessungen in schnellen, stromstarken Stoßentladungen
}

\author{
Von Günter Hofmann* \\ Aus dem Laboratorium für Technische Physik der Technischen Hochschule München \\ (Z. Naturforschg. 18 a, 309-317 [1963]; eingegangen am 13. November 1962)
}

\begin{abstract}
In hot dense plasmas-as in the case of shock discharges-LANGMUIR probe measurements are difficult because complete probe characteristics are mostly unobtainable. Therefore, this work shows the possibility of getting information about the plasma at low probe voltages.

It is shown that for a sufficiently small probe voltage the current drawn by a symmetrical plane double probe is proportional to $n_{\mathrm{i}} / \sqrt{T_{\mathrm{e}}}\left(n_{\mathrm{i}}=\right.$ ion density, $T_{\mathrm{e}}=$ electron temperature $)$.

Knowing the approximate value of the electron temperature, the probe current gives the ion density immediately. Also the directed motion of ions can be determined by changing the orientation of the probe.

A good time resolution $\left(10^{-7} \mathrm{sec}\right)$ was achieved by matching the impedance between the probe and the plasma to the coaxial probe circuit.

The usefulness of this probe technique was investigated in a linear shock discharge with a coaxially, circular, plane double probe. Ion densities up to $10^{15} \mathrm{~cm}^{-3}$ and directed ion velocities of some $10^{5} \mathrm{~cm} / \mathrm{sec}$ were observed.

Simultaneously the electrode voltage, current derivative, and the local azimuthal magnetic field in the plasma were measured.
\end{abstract}

Elektrische Sonden sind ein wichtiges Diagnostikmittel für die Untersuchung ionisierter Gase.

In der ältesten Form der Langmuinschen Einzelsonde (ES) wird einer kleinen Hilfselektrode im Plasma eine Spannung gegenüber einer räumlich weit entfernten Referenzelektrode aufgeprägt und der zur Hilfselektrode fließende Strom gemessen. Als Referenzelektrode dient dabei zumeist die Anode oder Kathode der stationären Entladung. Aus der Abhängigkeit des Sondenstromes von der Sondenspannung, der sog. Sondencharakteristik, lassen sich Größen wie die Elektronentemperatur, Elektronendichte, Plasma- und Wandpotential sowie die Geschwindigkeitsverteilung der Elektronen bestimmen.

In zeitlich schnell veränderlichen, stromstarken Stoßentladungen ist die ES im allgemeinen nicht mehr brauchbar. Der wesentliche Grund dafür ist, daß meist kein einfacher Zusammenhang mehr zwischen dem Potential des Plasmas am Ort der Sonde und am Ort der Referenzelektrode besteht. Des weiteren nehmen die maximalen Elektronen-Einströme zur Sonde, deren Bestimmung die ES-Methode erfordert, in dichten, heißen Plasmen so hohe Werte an, daß die Sonde thermisch zu stark belastet wird. Außerdem stören die starken Magnetfelder in Stoßentladungen die Messung beträchtlich, da bei der

\footnotetext{
* Auszug aus der von der Fakultät für Allgemeine Wissenschaften der Technischen Hochschule München genehmigten Dissertation.
}

ES-Methode die Elektronen die hauptsächlichen Sondenstromträger sind.

Diese Schwierigkeiten lasen sich z. Tl. durch die Anwendung der Technik "schwimmender" Doppelsonden (DS) vermeiden. Bei dieser Methode befinden sich zwei - meist flächengleiche - Sondenelektroden in so enger Nachbarschaft, daß das Plasma um beide Elektroden als einheitlich angesehen werden kann. Der Sondenkreis ist nicht an äußere Potentiale angekoppelt, dem Plasma wird deshalb kein Gesamtstrom entzogen.

Dies hat den Vorzug, daß das nichtstationäre Plasmapotential am Ort der DS ganz außer acht gelassen werden kann, da das Potential des Doppelsondenkreises dem Plasmapotential folgt.

Der maximale Strom im Sondenkreis, der bei einer wachsenden, eingeprägten EMK zwischen den Sondenelektroden fließt, ist durch den maximalen Ioneneinstrom zur Sonde gegeben. Dieser ist zwar größer als der thermische Ioneneinstrom, aber immer noch wesentlich kleiner als der thermische Elektroneneinstrom, wie er bei der ES gemessen wird.

Die Tatsache, daß der Strom wesentlich durch Ionen bestimmt wird, ist vor allem deshalb von Vorteil, weil die Ionen wegen ihrer größeren Masse vom Magnetfeld der Entladung sehr viel weniger

Diese Arbeit wurde im Rahmen des Vertrages zwischen dem Institut für Plasmaphysik $\mathrm{GmbH}$ und der Europäischen Atomgemeinschaft über die Zusammenarbeit auf dem Gebiete der Plasmaphysik durchgeführt. 
beeinflußt werden als die Elektronen. Auf die Theorie der DS wird hier nicht näher eingegangen, sie ist an anderer Stelle ausführlich dargestellt ${ }^{1}$.

Bei der vorliegenden Arbeit ist nur der Fall einer symmetrischen ebenen Doppelsonde wichtig, für den die ideale Charakteristik eine besonders einfache Form annimmt:

$$
I_{\mathrm{s}}=j_{\mathrm{i}} F \operatorname{tgh}\left[e V_{\mathrm{s}} /\left(2 k T_{\mathrm{e}}\right)\right] .
$$

$j_{\mathrm{i}}$ stellt die maximale Ionenstromdichte zu einer negativen Elektrode dar ${ }^{2}$ :

$$
j_{\mathrm{i}}=\exp (-0,5) \cdot e n_{\mathrm{i}} V k T_{\mathrm{e}} / M
$$

$I_{\mathrm{s}}=$ Sondenstrom,

$k=$ Boltzmann-Konstante,

$V_{\mathrm{s}}=$ Sondenspannung,

$M=$ Ionenmasse,

$F=$ Sondenfläche,

$T_{\mathrm{e}}=$ Elektronentemperatur,

$e=$ Elementarladung,

$n_{\mathrm{i}}=$ Ionendichte im ungestörten Plasma.

Aus der experimentell gewonnenen Sondencharakteristik lassen sich nach ${ }^{1}$, ähnlich wie bei der ES, die Elektronentemperatur und die Ionendichte ermitteln. Bei niedrigeren Elektronentemperaturen ist die DS-Methode sehr erfolgreich, jedoch tritt ab etwa $T_{\mathrm{e}}=70000^{\circ} \mathrm{K}$ die Erscheinung des sogenannten ,arcing“ auf, die die Aufnahme einer vollständigen Sondencharakteristik verhindert. Bei diesen hohen Temperaturen sind die Sondenspannungen, die für das Erreichen des Sättigungsteils der Charakteristik erforderlich sind, so hoch (über 15 Volt), $\mathrm{da}$ sich zwischen den Sondenelektroden eine Bogenentladung ausbildet, die meist die Sonde zerstört. Durch besondere Behandlung der Sondenelektroden läßt sich der Einsatz des Bogens nur wenig verschieben.

Ein unter Umständen brauchbarer Ausweg ist die Verwendung eines „Plasmaabschwächers“ 3 .

Die Sonde wird dabei in ein Rohr aus Isolationsmaterial versenkt, in das nun das Plasma diffundiert und an Dichte und Temperatur abnimmt. Als Folge davon rückt der Sättigungsknick der Sondencharakteristik zu kleineren Sondenspannungen hin und kann deshalb beobachtet werden. Die Plasmadaten außerhalb des Rohres müssen über eine Diffusionstheorie aus den am Ort der Sonde gemessenen Daten berechnet werden.

Abgesehen von den Unsicherheiten, die schon im stationären Fall dieser Extrapolation anhaften, werden die Verhältnisse hoffnungslos kompliziert, wenn

1 K.Y Амamoto u. T. OKUda, J. Phys. Soc., Japan 11, 57 [1956].

2 G. J. Schulz u. S. C. Brown, Phys. Rev. 98, 1642 [1955]. schnelle zeitliche Änderungen des Plasmas auftreten. In schnellen Stoßentladungen ist dieses Hilfsmittel unbrauchbar. Aus diesem Grunde wird untersucht, welche Aussagen über das Plasma schon bei niedrigen Sondenspannungen gemacht werden können.

\section{Reduziertes Verfahren zur Gewinnung der Ionendichte unter Berücksichtigung gerichteter Bewegungen der Ionen}

Bei einer ebenen, flächengleichen Doppelsonde wächst der Sondenstrom in der Umgebung des Nullpunktes der Charakteristik mit der Sondenspannung annähernd linear. Es ist daher sinnvoll, einen äquivalenten Plasmawiderstand $R_{0}=\left(\mathrm{d} V_{\mathrm{s}} / \mathrm{d} I_{\mathrm{s}}\right) V_{\mathrm{s}=0} \quad \mathbf{z u}$ definieren. Dieser ist nicht identisch mit dem elektrischen Widerstand eines Plasmas ohne Raumladungsschichten.

Der äquivalente Plasmawiderstand läßt sich sehr einfach aus (1) bestimmen:

$$
R_{0}=\frac{2 \exp (-0,5) \cdot \sqrt{M k T_{\mathrm{e}}}}{e^{2} F n_{\mathrm{i}}} .
$$

$R_{0}$ ist nur schwach von der Elektronentemperatur abhängig, dagegen umgekehrt proportional der Ionendichte. Wird die Elektronentemperatur nach anderen Methoden grob ermittelt, gibt $R_{0}$ die Ionendichte an.

$R_{0}$ kann sehr einfach gemessen werden. Es ist nur eine genügend kleine konstante Sondenspannung anzulegen, die noch im linearen Teil der Sondencharakteristik liegt. Dann ist der Sondenstrom $I_{\mathrm{s}}=V_{\mathrm{s}} / R_{0}$ direkt proportional der Ionendichte.

Fast stets ist jedoch mit hohen Plasmadichten und -temperaturen, bei denen das reduzierte Verfahren anzuwenden ist, auch eine gerichtete Bewegung der Ionen verbunden. Dies rührt einesteils vom Erzeugungsmechanismus her (Kompressionen), andernteils von starken Gradienten, z. B. der Dichte im Plasma. Aus diesem Grund muß der Einfluß einer gerichteten Bewegung der Ionen auf die Sondenmessung abgeschätzt werden.

Dabei wird der einfache Fall angenommen, daß die Größe $v_{\mathrm{r}}$ der gerichteten Bewegung der Ionen die mittlere thermische Geschwindigkeit der Ionen nicht wesentlich übersteigt. $v_{\mathrm{r}}$ sei die Komponente der gerichteten Bewegung senkrecht zur ebenen Sondenfläche. 
Es wird angenommen, daß sich dem Ioneneinstrom $j_{\mathrm{i}}$ an der Schichtgrenze ein Term $\Delta j_{\mathrm{i}}$ über. lagert, der der gerichteten Bewegung entspricht.

$$
\Delta j_{\mathrm{i}}=e n_{\mathrm{i}} v_{\mathrm{r}} .
$$

Die gesamte maximale Ionenstromdichte zur Sonde beträgt dann in Erweiterung von ( 1 a) :

$$
j_{\mathrm{i}}=e n_{\mathrm{i}}\left[\sqrt{\left(k T_{\mathrm{e}} / M\right)} \cdot \exp (-0,5)+v_{\mathrm{r}}\right] .
$$

Nach Einsetzen des Ausdrucks in (1) kann wieder der Sondenstrom für eine kleine Sondenspannung bestimmt werden:

$I_{\mathrm{s}}=\frac{V_{\mathrm{s}} F e^{2}}{2 k T_{\mathrm{e}}} n_{\mathrm{i}}\left[\sqrt{\frac{k T_{\mathrm{e}}}{M}} \cdot \exp (-0,5)+v_{\mathrm{r}}\right]$.

Die zusätzliche Abhängigkeit des Sondenstroms $I_{\mathrm{s}}$ von der Geschwindigkeit $v_{\mathrm{r}}$ setzt an sich die Kenntnis von $v_{\mathrm{r}}$ voraus, wenn $n_{\mathrm{i}}$ ermittelt werden soll oder umgekehrt. Durch passende Orientierung der Sonde kann aber erreicht werden, daß die Geschwindigkeit $v_{\mathrm{r}}$ einmal mit positivem und einmal mit negativem Vorzeichen eingeht. Dazu muß die Flächennormale der Sonde einmal entgegen der Ge. schwindigkeit $v_{\mathrm{r}}$ und einmal in ihre Richtung zeigen. Diese beiden Stellungen der Sonde seien mit den Indizes a bzw. b gekennzeichnet. $I_{\mathrm{sa}}$ bzw. $I_{\mathrm{sb}}$ seien die entsprechenden gemessenen Sondenströme.

Aus (3) ergibt sich dann sofort:

$$
\frac{I_{\mathrm{sa}}+I_{\mathrm{sb}}}{2}=\frac{V_{\mathrm{S}} F e^{2} \exp (-0,5)}{2 \sqrt{k} T_{\mathrm{e}} M} n_{\mathrm{i}} .
$$

Das Mittel der Meßwerte in den beiden Stellungen $\mathrm{a}$ und $\mathrm{b}$ eliminiert die gerichtete Bewegung $v_{\mathrm{r}}$ und ist identisch mit dem Sondenstrom im Fall $v_{\mathrm{r}}=0$. Der Ausdruck (4) wird später zur Ermittlung der Ionendichte $n_{\mathrm{i}}$ verwendet.

Andererseits läßt sich aus (3) bilden:

$$
\frac{I_{\mathrm{sa}}-I_{\mathrm{sb}}}{I_{\mathrm{sa}}+I_{\mathrm{sb}}}=\frac{\exp (-0,5)}{2} \cdot \sqrt{\frac{M}{k T_{\mathrm{e}}}} \cdot v_{\mathrm{r}} .
$$

Dieser Ausdruck, der als „relative Asymmetrie“ des Sondenstromes bezeichnet wird, ist im wesentlichen proportional zur gerichteten Bewegung der Ionen, die daraus bestimmt werden soll.

Die Sondenmessungen gestalten sich recht einfach, wenn die gerichtete Bewegung der Ionen zu einem Zentrum symmetrisch ist. Dann sind die beiden Stellungen a und b verwirklicht durch zwei Orte im gleichen Abstand vom Symmetriezentrum, wobei einmal die Sonde das Zentrum durchsetzt. In Abb. 1 ist die Anordnung bei Zylindersymmetrie dargestellt. Voraussetzung ist dabei, daß die Sonde in der Stellung $\mathrm{b}$ die Entladung nicht stört.

Bevor die Meßergebnisse an einer linearen Stoßentladung diskutiert werden, wird die technische Ausführung von Sonde und Meßkreis im einzelnen beschrieben.

\section{Ausführung von Sonde und Meßkreis}

Zur Abschirmung der starken Störfelder der Entladung ist ein koaxialer Aufbau der Sonde zweckmäßig. Die ebenen Sondenelektroden sind konzentrisch angeordnet; elektrische Felder und gerichtete Bewegungen der Ionen parallel zur Elektrodenoberfläche haben daher keinen Einfluß auf die Messungen. In Abb. 2 ist diese Sondenform dargestellt.

Der störende Einfluß der Randzonen der Raumladungsschichten ist am geringsten, wenn als ionensammelnde (negative) Elektrode die zentrale Kreisfläche verwendet wird. Das koaxiale Sondenrohr wird mit der Sondenspannungsquelle durch ein Koaxialkabel von gleichem Wellenwiderstand verbunden.

Alle Widerstände im Sondenkreis sind klein zu halten gegenüber dem äquivalenten Plasmawiderstand, der den Sondenstrom bestimmen soll.

Damit das Meßsignal durch Reflexionen nicht verfälscht wird, soll der äquivalente Plasmawiderstand in der Nähe des Wellenwiderstandes des koaxialen Aufbaues liegen. Für ein gegebenes Plasma ist damit die Sondenfläche $F$ festgelegt. Als Anpassungsbedingung

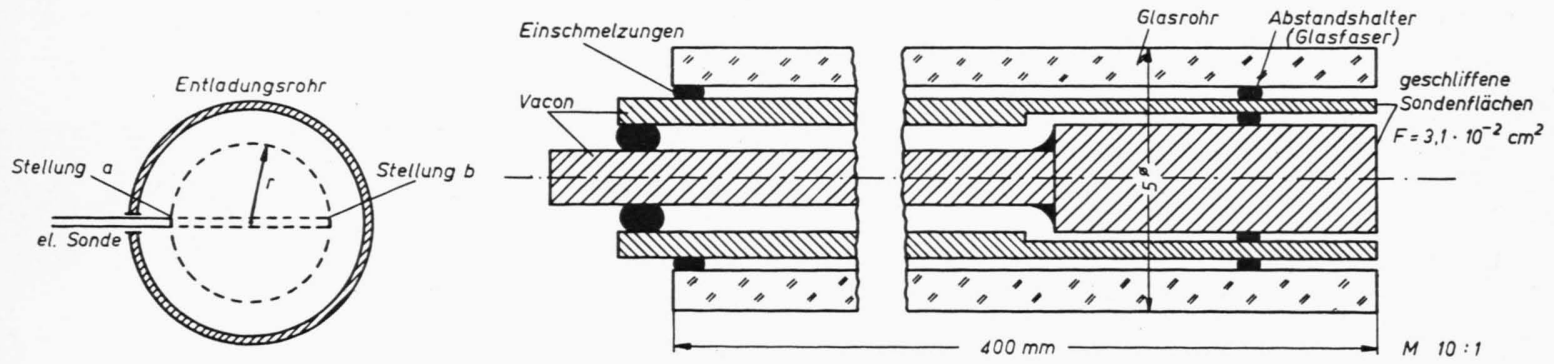

Abb. 1. Querschnitt durch das Entladungsrohr. Orientierung der Sondenelektroden a) zur Achse, b) zur Wand hin.

Abb. 2. Aufbau der koaxialen Doppelsonde. 


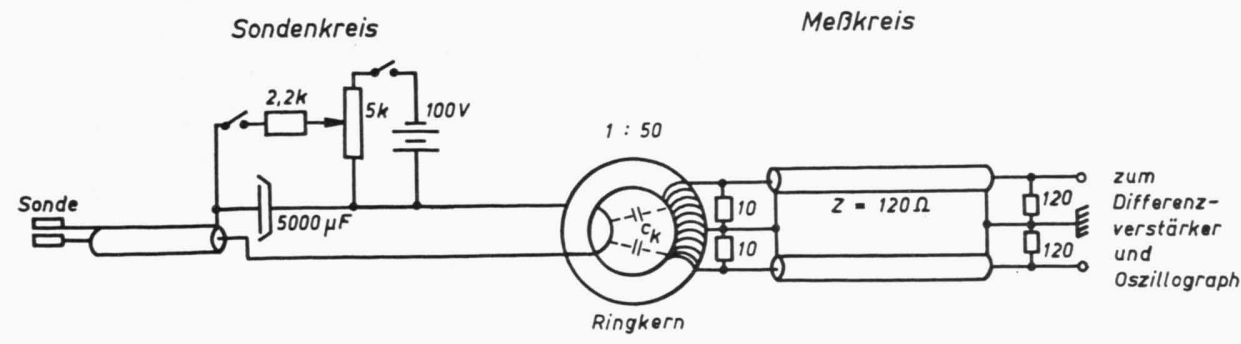

Abb. 3. Schaltung des Sondenstrom-Meßkreises.

ergibt sich somit der Radius $r_{\mathrm{s}}$ der zentralen Kreisfläche zu:

$$
r_{\mathrm{S}}=2,5 \cdot 10^{5}\left(\sqrt[4]{T_{\mathrm{e}} A} / \sqrt{n_{\mathrm{i}} Z}\right),
$$

$T_{\mathrm{e}}=$ Elektronentemperatur in ${ }^{\circ} \mathrm{K}, A=$ Atomgewicht der Ionen, $Z=$ Wellenwiderstand in $\mathrm{Ohm}, n_{\mathrm{i}}=$ Ionendichte in $\mathrm{cm}^{-3}$.

Mit einer so dimensionierten Sonde wurde eine Zeitauflösung von $10^{-7} \mathrm{sec}$ erreicht.

Ein weiteres wichtiges Problem ist die Messung des Sondenstromes ohne Beeinflussung des SondenkreisPotentials, das durch das Plasma bestimmt werden soll. Dies wurde erreicht durch Trennung des Meßkreises vom Sondenkreis mittels eines Stromwandlers. Dafür war ein hochpermeabler Ringkern aus Ferroxcube gut geeignet, der eine sehr geringe Streuung und eine niedrige Dämpfung aufwies.

Die primäre Wicklung im Sondenkreis bestand aus einer einzigen hochspannungsfesten Windung. Auf der Sekundärseite waren meist 50 Windungen angebracht, die den Meßwiderstand herabtransformierten.

Zwischen Primär- und Sekundärwicklung treten hohe Potentialdifferenzen auf. Wird auf der Sekundärseite asymmetrisch gemessen, so verursachen die Kopplungskapazitäten $c_{\mathrm{k}}$ zwischen den beiden Wicklungen starke Störungen. Diese können durch Symmetrierung der Sekundärwicklung eliminiert werden. Die Wicklungsmitte wird geerdet und die Signale der beiden Wicklungshälften einem Differenzverstärker zugeführt. Potentialverschiebungen zwischen Primär- und Sekundärwicklung geben nun Signale gleichen Vorzeichens und subtrahieren sich bei sorgfältiger Symmetrierung weg. Gemessen wird nur der Strom durch die Primärwicklung. In Abb. 3 ist das Prinzipschaltbild angegeben.

Mit dieser Anordnung wurden in einer linearen Stoßentladung Messungen gemacht, von denen im Abschn. 3 einige Beispiele gebracht werden.

\section{Beispiele von Sondenmessungen in einer linearen Stoßentladung}

Das Plasma wird erzeugt durch Entladung eines Kondensators über eine zylindrische Wasserstoffstrecke mit koaxialem Rückleiter.

Daten des Entladungskreises:

Länge des Entladungsrohres: $\quad 40 \mathrm{~cm}$,

Durchmesser des Entladungsrohres: $12 \mathrm{~cm}$,
Füllung: Wasserstoff von

Ladekondensator:

0,1 Torr,

Max. Ladespannung:

Max. Energieinhalt:

$7,7 \mu \mathrm{F}$

$18 \mathrm{kV}$,

1,25 kJoule.

In welchem Maße eine gerichtete Bewegung der Ionen auftritt, hängt von der Kompressionsgeschwindigkeit des Plasmas und damit von der dem Plasma zugeführten Leistung ab. Deshalb wurde die Entladung durch wahlweises Zuschalten einer Induktivität im Außenkreis verlangsamt.

\begin{tabular}{|l|c|l|}
\hline \multicolumn{1}{|c|}{ Entladung } & langsam & \multicolumn{1}{c|}{ schnell } \\
\cline { 1 - 1 } \begin{tabular}{l|c|} 
Entladungsfrequenz $f$ \\
Gesamtinduktivität $L$
\end{tabular} & 40 & $104 \mathrm{kHz}$ \\
max. Entladungsstrom $I_{\max }$ & 2030 & $324 \mathrm{~cm}$ \\
$\begin{array}{l}\text { max. Stromänderung } \\
(\mathrm{d} I / \mathrm{d} t)_{\max }\end{array}$ & $8,86 \cdot 10^{9}$ & $5,6 \cdot 10^{10} \mathrm{~A} / \mathrm{sec}$ \\
\hline
\end{tabular}

Die zeitliche Änderung des gesamten Entladungsstromes wurde mit einer bandförmigen RogowskiSpule aufgenommen, die Elektrodenspannung am Entladungsgefäß mit einem induktionsarmen Spannungsteiler. Das azimutale Magnetfeld $B_{\varphi}$ am Ort der elektrischen Sonde wurde mit einer kleinen Induktionsspule und nachgeschaltetem Integrationsglied gemessen. Die Spule war wie die elektrische Sonde radial verschiebbar und befand sich immer in einem Abstand von $0,5 \mathrm{~cm}$ vor der elektrischen Sonde. Durch Einzelmessungen wurde sichergestellt, da $\beta$ sich die beiden Sonden gegenseitig nicht stören. Als weiteres Diagnostikmittel sei noch eine elektrische Gabelsonde erwähnt, mit der nach einem Gegenspannungsverfahren die elektrische Längsfeldstärke im Plasma bestimmt wurde. (Siehe Abschn. 3.2.)

\subsection{Punktweise Aufnahme einer vollständigen Sondencharakteristik}

Bei niedriger Temperatur und Dichte des Plasmas (langsame Entladung von $40 \mathrm{kHz}$ und niedrige Ladespannung von $10 \mathrm{kV}$ ) liegt der Sättigungsknick der 
Sondencharakteristik noch bei genügend kleinen Sondenspannungen. Da außerdem die Reproduzierbarkeit der Entladung ausreichend war, konnte eine Sondencharakteristik punktweise durch Variation der Sondenspannung aufgenommen werden. Abb. 4 stellt ein Oszillogramm des Sondenstroms in der

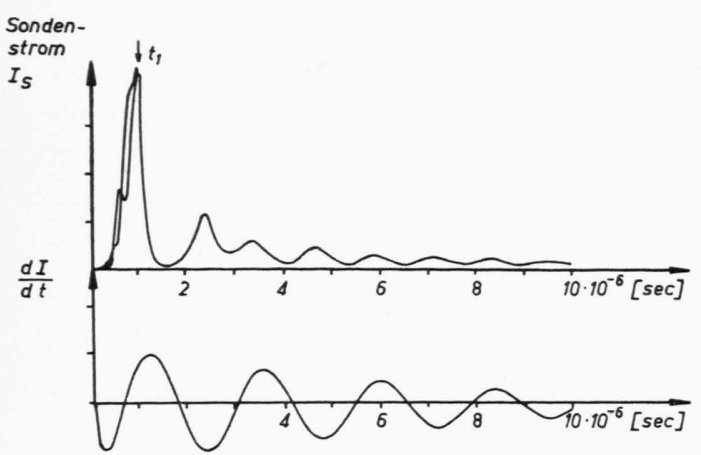

Abb. 4. Oben: elektrische Sondenmessung in der Entladungsachse. 2 Aufnahmen unter gleichen Anfangsbedingungen. Unten: zeitliche Änderung des gesamten Entladungsstromes.

Entladungsachse dar. Bei dieser Aufnahme war die Sondenspannung relativ niedrig und somit der Sondenstrom proportional der Ionendichte. Wie ersichtlich ist, stimmt der Verlauf des Sondenstroms im wesentlichen überein mit dem Verlauf des Quadrats des Entladungsstromes und damit der Leistung, die das Plasma aufnimmt. Diese Proportionalität zwischen Ionendichte und der vom Plasma aufgenom- menen Leistung zeigt, daß die aufgenommene Energie hauptsächlich zur Ionisierung des Gases verwendet wird.

Für den Zeitpunkt des Sondenstrommaximums $t_{1}$ ist als Beispiel die Charakteristik aufgezeichnet (Abb. 5). Die Charakteristik ist etwas unsymmetrisch, da die Fläche der äußeren Ringelektrode kleiner war als die der Zentralelektrode.

Die Auswertung nach ${ }^{1}$ ergab eine Ionendichte $n_{\mathrm{i}}=3,6 \cdot 10^{13} \mathrm{~cm}^{-3}$ und eine Elektronentemperatur $T_{\mathrm{e}}=69000^{\circ} \mathrm{K}$. Die Anfangsdichte des Neutralgases betrug $3,3 \cdot 10^{15} \mathrm{H}_{2}$-Moleküle $/ \mathrm{cm}^{3}$.

\subsection{Bestimmung der Ionendichte bei Abwesenheit einer gerichteten Ionenbewegung}

Wenn das Plasma stärker aufgeheizt wird (langsame Entladung von $40 \mathrm{kHz}$ und volle Ladespannung von $18 \mathrm{kV}$ ), dann rückt der Sättigungsknick der Sondencharakteristik zu hohen Sondenspannungen hin, bei denen Bögen zwischen den Sondenelektroden auftreten. Deshalb wird nach dem reduzierten Verfahren nur eine konstante, niedrige Sondenspannung $(7,5 \mathrm{~V})$ angelegt und aus dem dabei fließenden Sondenstrom die Ionendichte bestimmt. Abb. 6 zeigt eine Sondenmessung in der Entladungsachse während der ersten Halbwelle.

In einigem Abstand von der Achse zeigen die Sondensignale einen ganz anderen Verlauf (Abb. 7). Der plötzliche Sprung bei $4,5 \mu$ sec deutet auf ein Überstreichen der beiden Sonden durch eine strom-

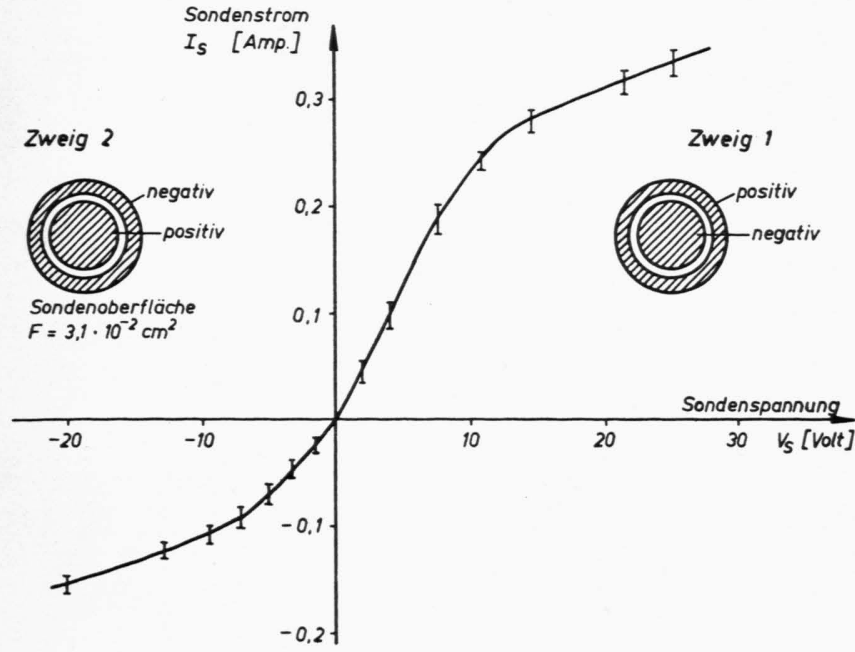

Abb. 5. Sondercharakteristik für den Zeitpunkt $t 1$ in der Entladungsachse.

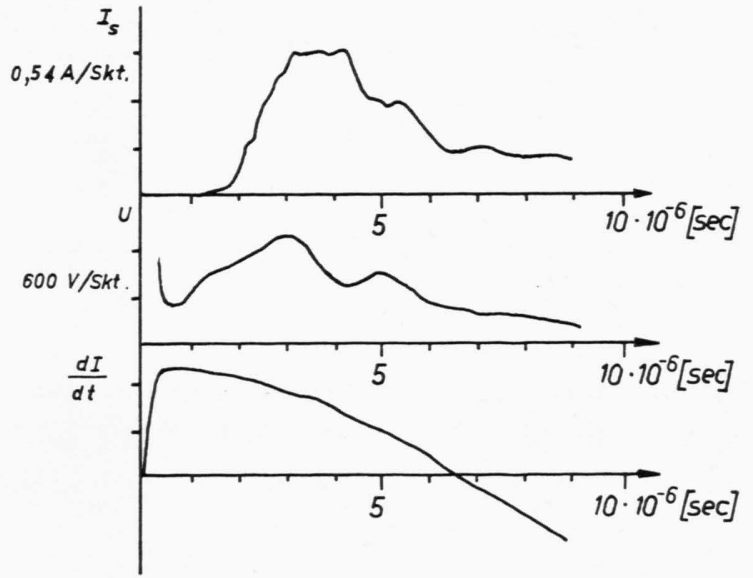

Abb. 6. Oben: elektrische Sondenmessung in der Entladungsachse. Mitte: Spannung zwischen den Gefäßelektroden. Unten: zeitliche Änderung des gesamten Entladungsstromes. 
führende Plasmaschicht hoher Ionendichte in Richtung zur Wand hin. Dies kann entweder ein asymmetrisches Ausbiegen der ganzen Plasmasäule oder

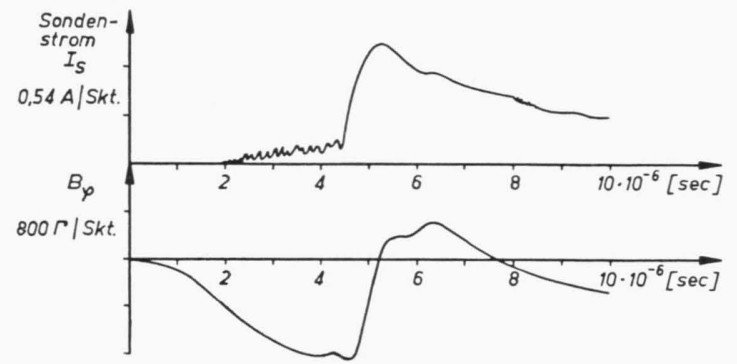

Abb. 7. Signale der elektrischen Sonde (oben) und der Magnetfeldsonde (unten) in $2 \mathrm{~cm}$ Abstand von der Entladungsachse.

eine symmetrische Expansion bedeuten. Aus spiegelbildlichen Messungen ergab sich, daß die Plasmasäule symmetrisch expandiert. Die Expansion ist so heftig, daß der Sprung in der Ionendichte noch weit im Sondenstutzen am Entladungsgefäß nachweisbar ist. Durch Umzeichnen lassen sich Momentaufnahmen des radialen Verlaufs des Sondenstroms gewinnen (Abb. 8).

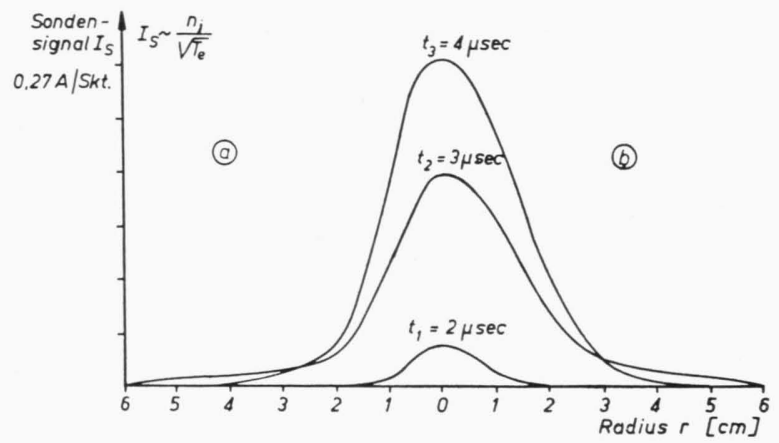

Abb. 8. Radialer Verlauf des Sondenstromes $I_{\mathrm{S}}$ zu verschiedenen Zeiten. Sondenspannung 7,5 V. Beim Teil b der Kurven durchsetzt die Sonde die Achse.

In Abb. 9 ist bereits die graphisch aus $\operatorname{dem} B_{\varphi^{-}}$Verlauf ermittelte Entladungsstromdichte $j_{z}$ aufgetragen. Für die Darstellung der Expansion war die Reproduzierbarkeit nicht ausreichend.

Aus den bisherigen Meßergebnissen läßt sich der Ablauf der Entladung während der ersten Halbwelle folgendermaßen deuten:

Infolge der niedrigen Entladungsfrequenzen und der fehlenden Vorionisierung bildet sich kein SkinEffekt aus; über den Querschnitt wird gleichmäßig ionisiert.
Das wachsende Eigenmagnetfeld beeinflußt die Elektronenbewegung vor allem in den äußeren Regionen, da es dort am stärksten ist. In der Achse nimmt die Ionisierung stärker zu als weiter außen $(2 \mu \mathrm{sec})$.

Abb. 9. Radialer Verlauf der Entladungs. stromdichte $j_{z}$ zu ver schiedenen Zeiten.

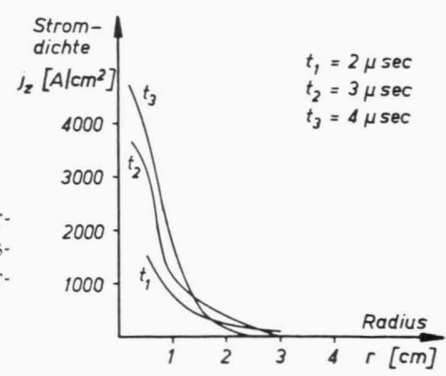

Eine radiale Expansion der inneren Regionen wird durch einen beginnenden Pinch-Effekt verhindert, der sogar eine leichte Kompression der ganzen Säule bewirkt $(4 \mu \mathrm{sec})$. Es bildet sich ein enger Kanal hoher Stromdichte in der Achse aus. In diesem Kanal übersteigt nun sehr rasch der gaskinetische Druck den Druck des Eigenmagnetfeldes, was zu einer heftigen Expansion der ganzen Plasmasäule nach $4,5 \mu$ sec führt.

Die Abb. 8 und 9 geben 3 Momentaufnahmen der Kompressionsphase wieder. Die Kompressionsgeschwindigkeit ist klein gegen die mittlere thermische Geschwindigkeit der Ionen, wie aus der Symmetrie der elektrischen Sondensignale folgt. Die Symmetrie ist außerdem ein Zeichen dafür, daß die elektrische Sonde die Entladung nicht stört, wenn sie die Achse der Entladung durchsetzt.

Aus dem Sondenstrom $I_{\mathrm{s}}$ in Abb. 8 geht die Ionendichte nicht unmittelbar hervor, da die Elektronentemperatur unbekannt ist. Bei dieser relativ langsamen Entladung besteht aber die Möglichkeit, aus der elektrischen Längsfeldstärke und der Entladungsstromdichte die Temperatur abzuschätzen. Während der langsamen Kompression verhält sich das Plasma im wesentlichen wie ein ohmscher Widerstand. Nun ist im allgemeinen der spezifische Widerstand $\varrho_{\mathrm{p}}$ eines Plasmas im Magnetfeld anisotrop. In der Entladungsachse ist jedoch das Magnetfeld Null, hier läßt sich einfach schreiben:

$$
\varrho_{\mathrm{p}}=E_{z} / j_{z},
$$

$E_{z}=$ elektrische Längsfeldstärke, $j_{z}=$ axiale Entladungsstromdichte.

Der spezifische Widerstand eines vollionisierten Plasmas ist in erster Linie nur von der Elektronentemperatur abhängig ${ }^{4}$. Mit der gemessenen elektri- 
schen Feldstärke und der Stromdichte wäre somit die Temperatur ermittelt. Bei der vorliegenden Entladung ist aber wahrscheinlich das Plasma nicht vollionisiert. Der Einfluß des restlichen Neutralgases auf den spezifischen Widerstand ist deshalb mit zu berücksichtigen. Da jedoch die Neutralgasdichte während der Entladung unbekannt ist, wurde ein Ansatz mit maximalem Einfluß des Neutralgases gemacht. Die Ergebnisse sind in Tab. 1 dargestellt.

\begin{tabular}{|c|c|c|c|}
\hline & $t_{1}=2 \mu \mathrm{sec}$ & $t_{2}=3 \mu \mathrm{sec}$ & \multicolumn{1}{|c|}{$t_{3}=4 \mu \mathrm{sec}$} \\
\hline$n_{\mathrm{e}}$ & $3,3 \cdot 10^{13}$ & $1,9 \cdot 14$ & $3,9 \cdot 10^{14} \mathrm{~cm}^{-3}$ \\
$T_{\mathrm{e}}$ & 33800 & 40000 & $71000{ }^{\circ} \mathrm{K}$ \\
\hline
\end{tabular}

Tab. 1. Elektronendichten und -temperaturen in der Entladungsachse bei maximaler Abschätzung des Neutralgases.

Bei vollständiger Vernachlässigung des Neutralgases ergeben sich nur zur Zeit $t_{1}=2 \mu$ sec Abweichungen $\left(T_{\mathrm{e}}\right.$ um $30 \%, n_{\mathrm{i}}$ um $10 \%$ niedriger). Das bedeutet, daß zu den beiden späteren Zeiten der Ionisierungsgrad so hoch ist, daß der spezifische Widerstand nur durch das Plasma bestimmt wird.

Eine Fehlerabschätzung zeigt, daß in die Berechnung der Temperatur vor allem die elektrische Feldstärke eingeht, während auf die Ionendichte der äquivalente Plasmawiderstand den größten Einfluß hat.

Die elektrische Feldstärke ergab sich aus der Elektrodenspannung und dem Abstand der Elektroden. Diese so erhaltene mittlere Feldstärke kann von der am Ort der Sonde tatsächlich vorhandenen abweichen. Aus diesem Grund wurde zusätzlich die örtliche Feldstärke in der Achse direkt gemessen mit einer "schwimmenden“ elektrischen Gabelsonde. Beı dieser Messung sind Radialbewegungen des Plasmas ohne Einfluß. Im Rahmen der Meßgenauigkeit konnte kein Unterschied zwischen der mittleren Feldstärke und der örtlichen Feldstärke gefunden werden, eine Folge der nur schwachen Kompressionsbewegung. Da das angewandte Gegenspannungsmeßverfahren sich für schnelle Stoßentladungen gut eignete, wird es am Ende dieses Kapitels näher beschrieben.

Wegen des induktiven Anteils $L(\mathrm{~d} I / \mathrm{d} t)$ der elektrischen Feldstärke ist diese höher als es dem rein ohmschen Plasmawiderstand entspricht. Die wahren Werte der Temperatur liegen deshalb etwa 25\% höher als in Tab. 1 angegeben.

${ }^{4}$ L. Spitzer JR., Physics of Fully Ionized Gases, Interscience Publishers Ltd., London 1956, S. 84.
In den äquivalenten Plasmawiderstand geht der maximale Ioneneinstrom zu einer negativen Elektrode ein ( 1 a). Die Theorien darüber weichen stark voneinander $a b$, eine experimentelle Entscheidung steht noch aus ${ }^{5}$. Dadurch erhält der Absolutwert der Ionendichte in Tab. 1 eine Unsicherheit von etwa $50 \%$.

$$
\begin{gathered}
\text { Feldstärkemessung mit einer Gabel- } \\
\text { sonde }
\end{gathered}
$$

Die Gabelsonde war identisch mit der in Abb. 2 gezeigten ebenen Sonde; es wurden auf die Elektroden der ebenen Sonde zwei dünne, parallele Drähtchen aufgeschweißt und die Stirnfläche daraufhin mit Araldit überzogen (Schaltung wie in Abb. 3).

Eine direkte Spannungsmessung mit hochohmigem Sondenkreis (hochohmig gegenüber dem äquivalenten Plasmawiderstand) erwies sich wegen des Auftretens sehr starker Schwingungen als unmöglich. Deshalb wurde eine Strommessung vorgenommen, bei der der Sondenkreis niederohmig gegen den äquivalenten Plasmawiderstand gehalten werden kann. In den Sondenkreis wird eine feste Gegenspannung gelegt, die der Potentialdifferenz zwischen den Sondendrähtchen im Plasma entgegenwirkt. Der Sondenstrom läßt sich sehr leicht messen. Es sind diejenigen Zeitpunkte auf dem Sondenstromoszillogramm aufzusuchen, zu denen der Sondenstrom Null ist. $\mathrm{Zu}$ diesen Zeiten ist die Gegenspannung gleich der Potentialdifferenz zwischen den Sondenelektroden im Plasma. Die Feldstärke ergibt sich aus dem Abstand der parallelen Sondendrähtchen. Abb. 10 zeigt ein Meßbeispiel. Der zeitliche Verlauf der Feldstärke wird durch Variation der Gegenspannung gewonnen.

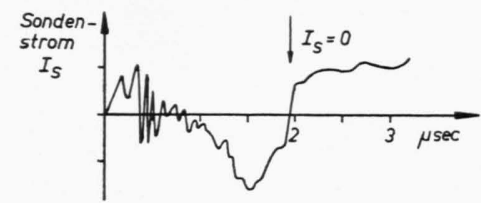

Abb. 10. Örtliche Feldstärkemessung mit einer Gabelsonde. Tendenz der Gegenspannung (22,5 V) : $\uparrow$. Tendenz der Feldstärke im Plasma: $\downarrow$. Zur Zeit $t_{I_{\mathrm{S}}=0}$ ergab sich eine Längsfeldstärke von 112,5 V/cm in der Achse.

\subsection{Bestimmung von Dichte und gerichteten Bewegungen der Ionen}

Wenn die Kondensatorenergie dem Plasma in kürzerer Zeit zugeführt wird (schnelle $104 \mathrm{kHz}$ Entladung, Ladespannung $18 \mathrm{kV}$ ), treten starke radiale Plasmabewegungen auf.

Der Sondenstrom, der nach dem reduzierten Verfahren bei einer kleinen, konstanten Sondenspannung $(7,5 \mathrm{~V})$ gemessen wird, zeigt deshalb eine

5 E. Hayess u. K. Rademacher, Ann. Phys., Lpz. 8, 158 [1961]. 
starke Abhängigkeit von der Orientierung der Sondenelektroden (Abb. 11). Der Magnetfeldverlauf blieb in den beiden Stellungen a und b bis auf eine Vorzeichenumkehr derselbe.
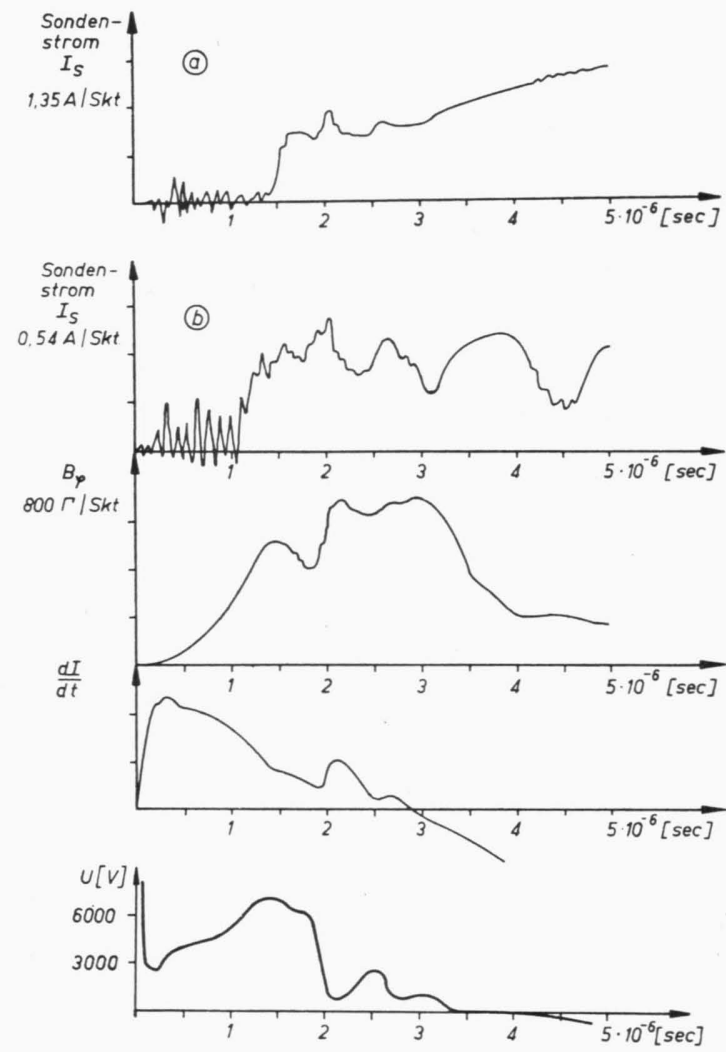

Abb. 11. Beispiel einer Sondenmessung in 2,5 cm Abstand von der Achse. Von oben nach unten: elektrische Sonde zur Achse orientiert, elektrische Sonde zur Wand orientiert, Magnetfeldsonde, zeitliche Änderung des Entladungsstromes, Elektrodenspannung am Entladungsgefäß.

Für 3 charakteristische Zeiten der Entladung wurden Momentaufnahmen des radialen Verlaufs des Sondenstromes $I_{\mathrm{s}}$ gezeichnet (Abb. 12) und nach den Gln. (4) und (5) die Ionendichte (Abb. 13) und die gerichtete Bewegung der Ionen (Abb. 14) daraus ermittelt.

Zur Zeit $t=1,5 \mu$ sec zeigt die Elektrodenspannung das erste Maximum, die Plasmasäule wird zum ersten Mal komprimiert. Innerhalb eines Radius von $2 \mathrm{~cm}$ läuft das Plasma nach innen, während es weiter außen bereits zur Wand strömt. Die größte Ionendichte liegt bei $r=2 \mathrm{~cm}$.

Zur Zeit $t=3 \mu \mathrm{sec}$ hat der Entladungsstrom seinen höchsten Wert erreicht, die zeitliche Stromänderung ist nahezu Null. Das Plasma ist im Zentrum
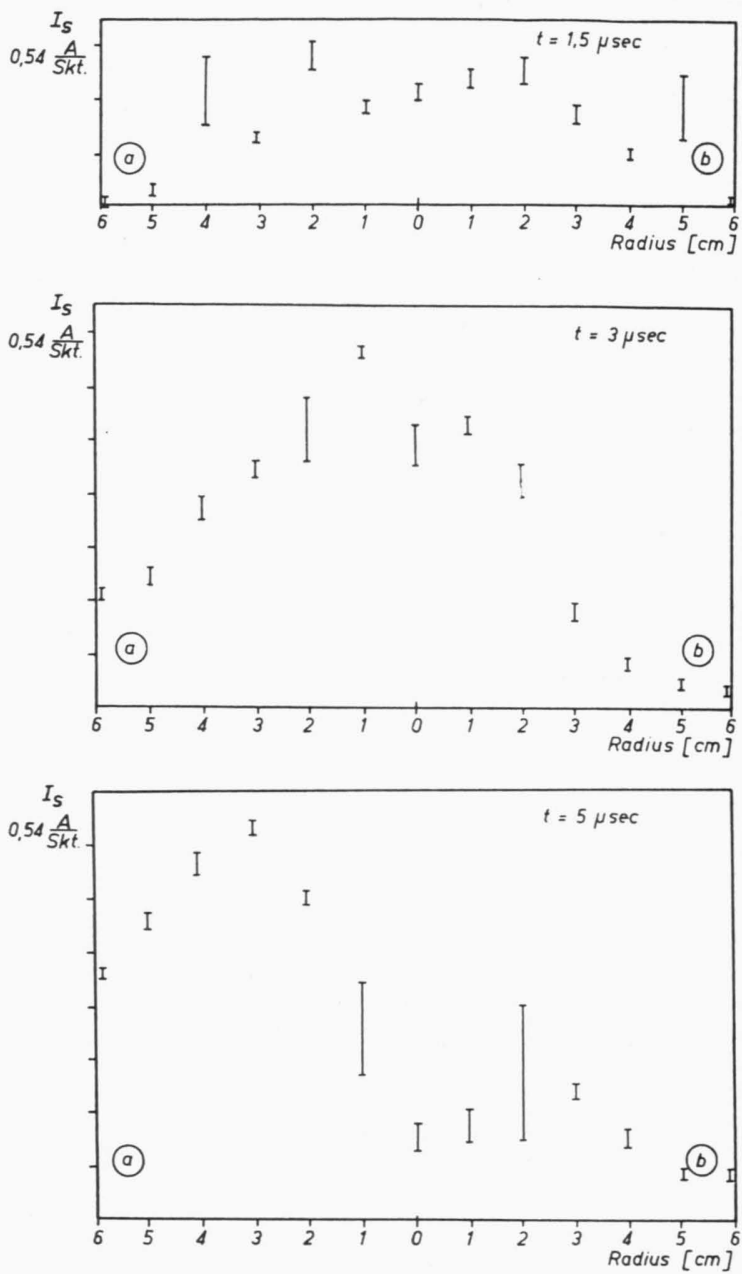

Abb. 12. Radialer Verlauf des Sondenstroms $I_{\mathrm{s}}$ zu 3 charakteristischen Zeiten der Entladung. Streuung von je 3 Aufnahmen unter gleichen Anfangsbedingungen.

fast in Ruhe; in den äußeren Bereichen strömt es zur Wand hin, wobei die Geschwindigkeit nach außen zunimmt. Die Plasmasäule ist stark kontrahiert mit einer hohen Ionendichte in der Achse. Das Dichtemaximum liegt jedoch etwas außerhalb der Achse bei $r=1 \mathrm{~cm}$. Das Maximum der Entladungsstromdichte liegt in der Achse.

Wenn später $(t=5 \mu \mathrm{sec})$ das komprimierende Magnetfeld verschwindet (Entladungsstrom geht durch Null), strömt das Plasma auch aus der Achse zur Wand hin. Die Ionendichte nimmt in der Achse stark ab, das Dichtemaximum liegt bei $r=3 \mathrm{~cm}$.

Die Absolutwerte von $n_{\mathrm{i}}$ und $v_{\mathrm{r}}$ können nicht genau angegeben werden, da die Elektronentemperatur unbekannt ist. Aus einem Vergleich mit den im Abschn. 3.2 errechneten Werten ergibt sich, daß die 
Elektronentemperatur von $100000{ }^{\circ} \mathrm{K}$ im Rahmen der nötigen Genauigkeit nicht allzu sehr verschieden ist. In den Abb. 13 und 14 sind die Werte von $n_{\mathrm{i}}$ und $v_{\mathrm{r}}$ angegeben, die einer Elektronentemperatur von $100000^{\circ} \mathrm{K}$ entsprechen.
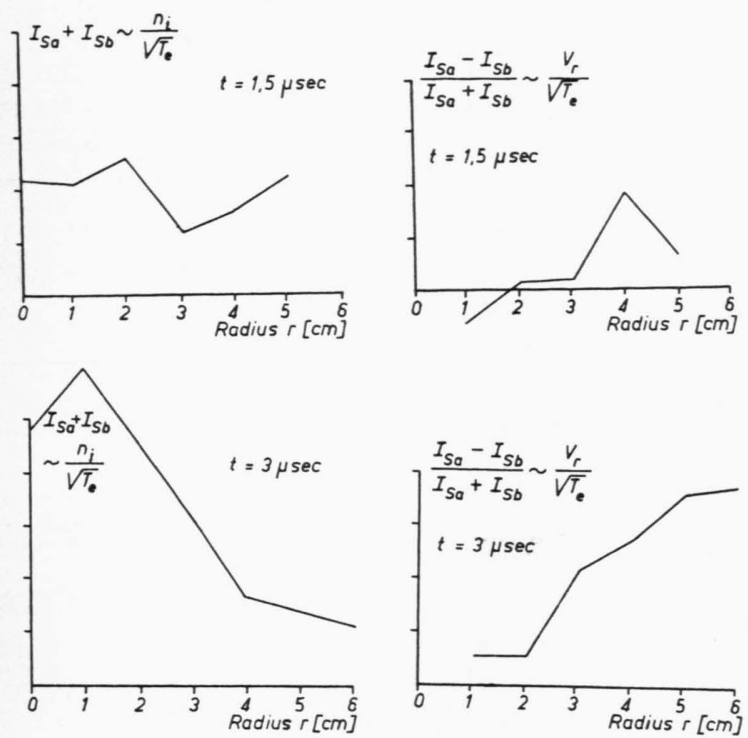

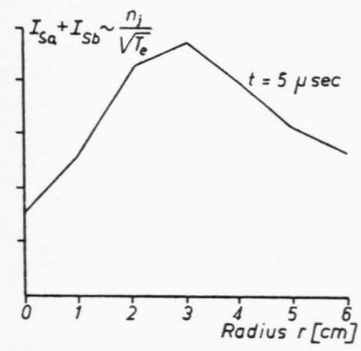

Abb. 13.

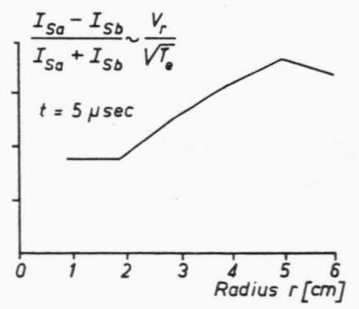

Abb. 14 .
Abb. 13. Mittel des Sondenstromes als Maß für die Ionendichte. Bei einer Elektronentemperatur von $100000{ }^{\circ} \mathrm{K}$ entspricht ein Skalenteil $1,4 \cdot 10^{14}$ Ionen $/ \mathrm{cm}^{3}$.

Abb. 14. Relative Asymmetrie des Sondenstroms als Maß für die gerichtete Bewegung der Ionen. Bei einer Elektronentemperatur von $100000{ }^{\circ} \mathrm{K}$ entspricht ein Skalenteil $3,5 \cdot 10^{5} \mathrm{~cm} / \mathrm{sec}$.

\section{Diskussion}

Elektrische Sonden lassen sich auch in dichten, heißen Plasmen schneller Stoßentladungen verwenden, wenn auf hohe Sondenspannungen und damit auf die Aufnahme vollständiger Sondencharakteristiken verzichtet wird. Das bedeutet eine Beschränkung der Messungen auf die Ermittlung des äquivalenten Plasmawiderstandes $R_{0}=\left(\mathrm{d} V_{\mathrm{s}} / \mathrm{d} I_{\mathrm{s}}\right)_{V_{\mathrm{s}}=0}$. Der Informationsgehalt dieses Wertes ist zwar kleiner als der einer vollständigen Sondencharakteristik, doch lassen sich daraus Ionendichte und gerichtete Bewegungen der Ionen bestimmen, wenn die Elektronentemperatur einigermaßen bekannt ist.

Bei Abweichungen von einer Maxwell-Verteilung der Elektronen ist bei der Doppelsonde die Temperatur der höher energetischen Gruppe maßgebend. Zur Ionendichte tragen dagegen auch die energieärmsten Ionen bei, die Sondenmessung gibt deshalb die wahre Ionendichte des Plasmas an.

Durch koaxialen Aufbau des Sondenkreises und Anpassung der Sonde an das Plasma kann eine hohe Zeitauflösung erreicht werden $\left(10^{-7} \mathrm{sec}\right)$. Die obere physikalische Grenze der Zeitauflösung ist durch die Trägheit des Aufbaues einer quasistationären Raumladungsschicht gegeben. Für ein Plasma von $n_{\mathrm{i}}=10^{14} \mathrm{~cm}^{-3}$ und $T_{\mathrm{e}}=4 \cdot 10^{4}{ }^{\circ} \mathrm{K}$ liegt diese Zeit etwa bei $10^{-10} \mathrm{sec}$, somit unterhalb der Zeitauflösung des elektronischen Meßkreises.

Das Magnetfeld der Entladung hat auf die Sondenmessung auch dann einen Einfluß, wenn der Ionengyroradius die Dicke der Raumladungsschicht vor den Sondenelektroden wesentlich übersteigt, wie es bei allen Experimenten der Fall war. Zur Sonde stellt sich ein Diffusionsstrom der Ionen ein, der zur Aufrechterhaltung einer stabilen Raumladungsschicht notwendig ist. Das Magnetfeld beeinflußt diese Diffusion sogar, wenn es parallel dazu orientiert ist ${ }^{6}$.

Die Sekundäremission von Elektronen aus den Sondenelektroden hat zur Folge, daß die Potentialdifferenz zwischen Elektroden und Plasma infolge teilweiser Kompensation positiver Raumladungen verändert wird. Da dieser Effekt aber bei beiden Elektroden in derselben Richtung auftritt, ist sein Einfluß im Vergleich zur Einzelsonde weniger stark.

Entgegen den wirklichen Verhältnissen wurde der Schatteneffekt der Sonde bei der Bestimmung von gerichteten Bewegungen der Ionen vernachlässigt. Das Umströmen des Plasmas um die Sonde erfordert eine genauere Betrachtung.

Es ist mir eine angenehme Pflicht, allen zu danken, die mich in dieser Arbeit unterstützten:

Herrn Prof. H. Maier-Leibnitz für die Aufnahme in das Laboratorium für Technische Physik, Herrn Prof. E. FünfER für die Stellung des Themas und Gewährung eines Arbeitsplatzes im Institut für Plasmaphysik, München-Garching, und Herrn Dr. W. Köppendörfer für förderliche Diskussionen.

${ }^{6}$ B. Bertotti, Phys. Fluids 4, 1047 [1961]. 